\title{
Dynamic lipidomic insights into phosphatidylcholine synthesis from organelle to organism
}

\author{
Alan N. Hunt* and Anthony D. Postle \\ Allergy and Inflammation Research, Division of Infection, Inflammation \& Repair, School of Medicine, \\ University of Southampton, Southampton SO16 6YD, United Kingdom
}

\begin{abstract}
Recent technical improvement and technological innovation in small molecule mass spectrometry have provided powerful tools for the intensive metabolomic biochemical investigations that will necessarily characterise the "post-genomic" era of biomedical research. For membrane phospholipids, use of tandem electrospray ionisation mass spectrometry (ESIMS/MS) exploiting precursor scanning of class-specific diagnostic fragments, can provide detailed quantitative profiles at the level of individual molecular species for many hundreds of unique lipids. Such "snapshot" measurements provide little information concerning metabolic flux. However, recent use of metabolic labelling with stable isotope derivatives of phospholipid headgroups combined with precursor scans of unlabelled and labelled fragments have yielded considerable insight into phosphatidylcholine metabolism in vivo. Here, we briefly review some of the recent work on pathways of phosphatidylcholine metabolism ranging from studies at subcellular organelle level through to whole organism. The sensitivity, specificity and suitability of this powerful methodological approach to numerous questions of phospholipid metabolism place ESI-MS/MS at the very heart of dynamic lipidomics in the foreseeable future.
\end{abstract}

Keywords: Electrospray ionisation mass spectrometry, phospholipid, phosphatidylcholine, molecular species, lipidomics, stable isotope

\section{Phosphatidylcholine (PtdCho) composition}

For the majority of almost 160 years since "lecithin" (phosphatidylcholine, PtdCho) was first recognised as phosphorus-containing lipid and substantial biological membrane component [16], methods for its detailed characterisation have remained essentially unchanged, aside from improvements in sensitivity. So, chromatographic separation following organic solvent extraction of biological samples has relied upon column chromatography or thin layer chromatography on a variety of matrices together with tailored solvent systems aimed at resolving the PtdCho class of glycerophospholipids from other glycerophospholipids on the basis of differences in the electrostatic properties of the lipid headgroup.

For purified PtdCho, modification of high performance TLC systems exploiting silver ions in argentation TLC permitted resolution of lipids with the same headgroup according to the number of unsaturated double bonds present at the $s n-1$ and $s n-2$ positions (Fig. 1). Advances in, and refinement of, high performance liquid chromatography (HPLC) techniques have allowed even greater resolution of PtdCho

\footnotetext{
${ }^{*}$ Corresponding author: Dr Alan N. Hunt, Mailpoint 803, Level F, South Lab and Path Block, Southampton General Hospital, Tremona Road, Southampton SO16 6YD, United Kingdom. Tel.: +44 (0)2380 794178; Fax: +44 (0)2380 796378; E-mail: anh@ soton.ac.uk.
} 


\section{Molecular species of phosphatidylcholines}

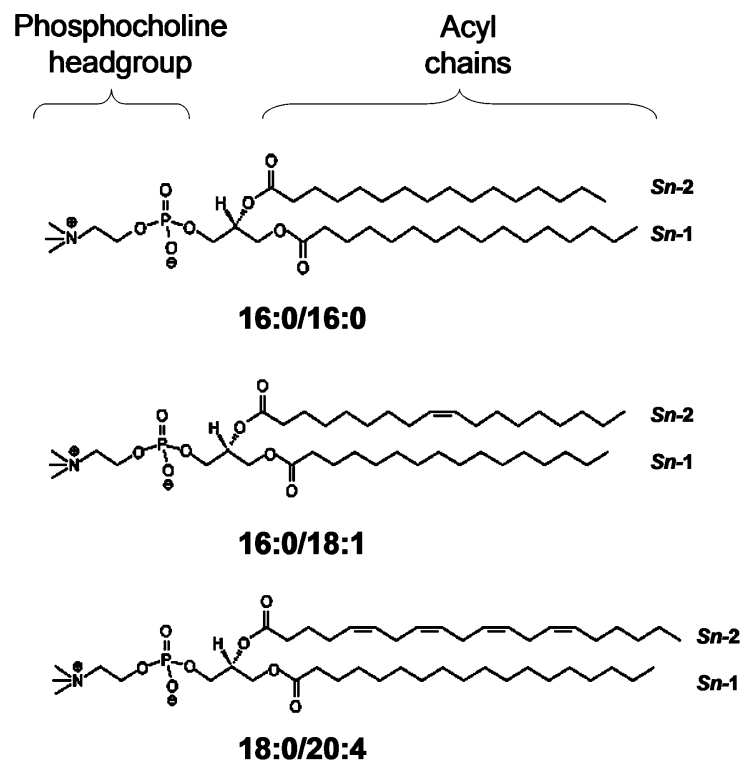

Fig. 1. Molecular species of phosphatidylcholines. The class of phospholipid is determined by the alcohol group joined to the glycerophosphate backbone which, in the case of PtdCho is choline. The molecular species is defined by the radyl chains - in these cases diacyl - attached to the $s n-1$ and $s n-2$ positions on glycerophosphate.

molecular species that vary by as little as a single double bond. However, in terms of detection and quantitation, the lack of an easily quantifiable physical property is problematic necessitating resort either to chemical destruction and subsequent analyses of, for example, inorganic phosphate or to complex chemical derivatisations which are both laborious and prone to systematic error. Post-column fluorescence detection in the presence of 1,6-diphenyl-1,3,5-hexatriene allowed dramatic increases in detection sensitivity $[2,9,23,29]$ but was still limited by a biophysical requirement of the lipid to be present in sufficient quantities to form vesicles into which the fluorophore could partition and thence fluoresce.

Advances in small molecule mass spectrometry in association with "soft" ionisation techniques since the early 90 s have rendered Tandem Electrospray Ionisation Mass Spectrometry (ESI-MS/MS) the ideal successor technology for phospholipid analysis and characterisation as explained in excellent recent reviews [17,30]. The ease of ionising the zwitterionic choline headgroup of PtdCho makes it a particularly good molecule to analyse under positive ionisation condition. However, when precursor scans of the fragmented phospholipid in positive ionisation and the diagnostic fragment of PtdCho with a mass/charge ( $\mathrm{m} / z$ ) ratio of $184^{+}$are employed (Fig. 2) the discrimination, sensitivities and specificities are obtained which are orders of magnitude greater than conventional methods. Indeed, theoretically, if not practically, every fragmented PtdCho molecule may be capable of detection limited only by the mass spectrometer's performance characteristics. For biomembrane samples, inclusion of "non-physiological" PtdCho molecular species internal standards and prior calibration of the instrument's response characteristics $[14,20,21,24]$ allow accurate estimation of the concentrations of individual molecular species.

Useful though quantitation of the absolute tissue concentrations or proportions of molecular species of PtdCho undoubtedly are for biological systems, they necessarily reflect a specific time point analysis. They do not inform the metabolic pathways and flux responsible for their generation even when conducted at physiological extremes. For example, analysis of cells grown under control conditions when 


\section{Structural Fragmentation of Endogenous and Deuterated PtdCho during ESI-MS/MS}

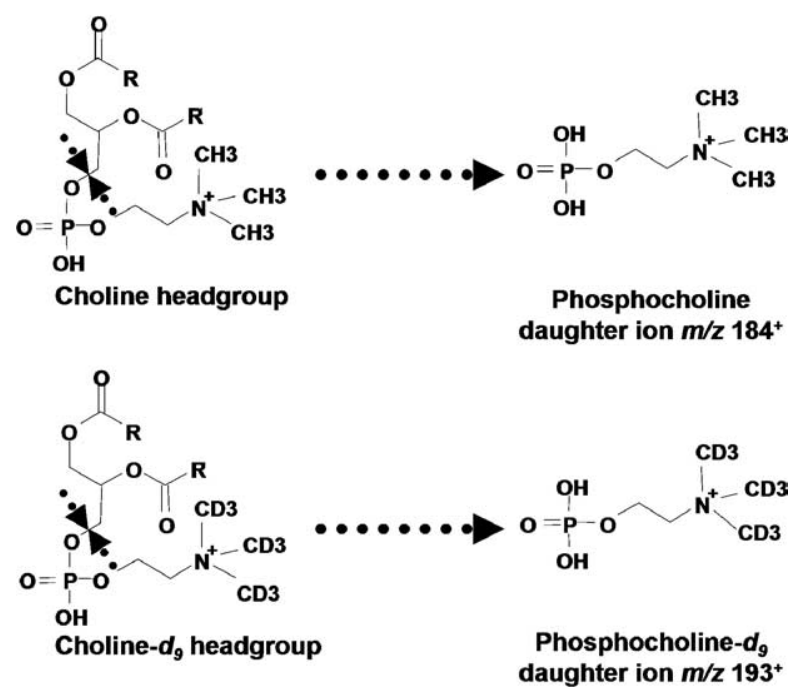

Fig. 2. Diagnostic fragmentation of phosphatidylcholine. The fragmentation of PtdCho under positive ionization conditions in ESI-MS/MS yields a characteristic fragment with an $m / z$ of $184^{+}$as shown. When choline- $d_{9}$ has been incorporated as the PtdCho headgroup, the increase in mass of 9 deuterons across the methyl groups yields a corresponding diagnostic fragment of $m / z 193^{+}$.

compared with those cultured for several days [21] or even weeks with polyunsaturated fatty acids [25] may reveal "end point" differences but only provide insight into the underlying mechanisms producing them with stable isotope labelling [21].

Historically, use of radionuclide-labelled precursors was the method of choice for tracking biomolecules through metabolic transformations. Use of radionuclides to follow labelled choline headgroups through PtdCho molecular species post HPLC using a flow through radiochemical detection system was a less than ideal option since it was expensive and, even with high specific activities, detection was limited not just by small incorporations but also by the decay rate of $\left[{ }^{3} \mathrm{H}\right]$ or $\left[{ }^{14} \mathrm{C}\right]$ and efficiency of counting [7,8]. Once again, ESI-MS/MS provides a neat alternative route which is at once more sensitive, more specific and above all much cheaper allowing near saturation of systems with stable isotopes $[20,21,24]$. The availability of stable isotope labelled choline headgroups, most notably choline- $d_{9}$, choline- $d_{13}$, and $\left[{ }^{13} \mathrm{C}\right]$-labelled alternatives provide, in conjunction with precursor scanning techniques, a powerful solution. The possibility of simultaneous determination of unlabelled PtdCho species using $m / z 184^{+}$precursors and, respectively, $m / z 193^{+}$precursors for choline- $d_{9}, m / z 197^{+}$precursors for choline- $d_{13}$ and $m / z 189^{+}$precursors for choline containing five ${ }^{13} \mathrm{C}$ molecules expanded metabolic flux determinations dramatically.

The stable isotope labelled fraction effectively represents newly synthesised PtdCho when the label is at saturating concentrations while the $m / z 184^{+}$precursors represents "endogenous" PtdCho as shown diagrammatically (Fig. 3). We have termed these studies "dynamic lipidomics" to distinguish them from the more widely employed static measurements $[17,30]$ and to describe an emerging technology that will likely dominate phospholipids metabolism in the coming years. Some examples of the applicability of stable isotope labelling to subcellular, whole cell and whole organism studies are given in Table 1 and 


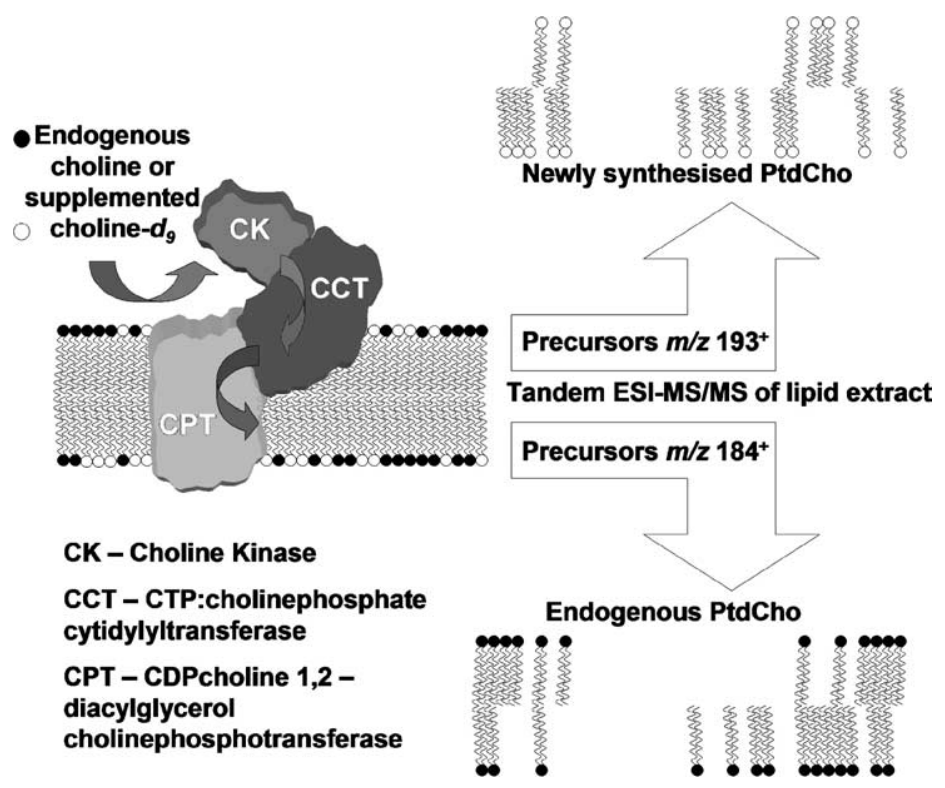

Fig. 3. The principle of stable isotope labeling of PtdCho with choline- $d_{9}$. Standard incorporation of choline via the CDPcholine pathway, represented by closed circles, yields a diagnostic fragment with $m / z 184^{+}$the can be used in precursor scans to reconstruct the parent molecule spectrum of PtdCho. Swamping the system with choline- $d_{9}$ redirects synthesis to incorporate this headgroup, represented by open circles, and the diagnostic fragment with $m / z 193^{+}$is then similarly used to reconstruct the parent molecule spectrum of newly synthesized PtdCho.

Table 1

Biological applications of deuterium stable isotope labelling and ESI-MS/MS analyses of phosphatidylcholine metabolism

\begin{tabular}{lll}
\hline Experimental system & Stable isotope label(s) & References \\
\hline Rat hepatocytes & $\begin{array}{l}\text { choline- } d_{9} \text {, ethanolamine- } d_{4} \\
\text { choline- } d_{9} \\
\text { choline- } d_{9}\end{array}$ & {$[14]$} \\
Human hepatocarcinoma & & {$[13]$} \\
IMR-32 neuroblastoma, HaCaT keratinocytes & choline- $d_{9}$ & {$[20,21,24]$} \\
HL60 cells, U937 cells and nuclei & choline- $d_{9}$ & {$[22]$} \\
CHO-K1 cells and hCEPT1 over-expressors & choline- $d_{9}$ & {$[19]$} \\
Mouse embryonic fibroblasts and nuclei & S $\left[\right.$ methyl- $d_{3}$ ]adenosyl-L-methionine, & {$[28]$} \\
Isolated human neutrophils and lymphocytes & choline- $d_{13}$, ethanolamine- $d_{4}$ & {$[4-6]$} \\
Saccharomyces cerevisiae & choline- $d_{9}$, ethanolamine- $d_{4}$ & {$[15]$} \\
choline- $d_{9}$ & {$[27]$} \\
Plasmodium falciparum & Whole human &
\end{tabular}

further elaborated below. The relatively recent application of these technologies mean that a number of the reports are still of preliminary nature. However, an explosion of interest in the techniques seen in many abstracts at bioscience meetings will likely see many more in the coming years.

\section{Tracking deuterated choline flux through defined biological systems}

The recognition that subcellular membranes contain different proportions of individual molecular species of PtdCho is not new and it is well established that plasma membrane PtdCho composition [31] 
differs from that of the endoplasmic reticulum [32] which in turn varies from that of the nuclear envelope $[33,35]$ and those membrane lipids that colocalise with the matrix of the nucleus [20,21]. However, until the mass spectrometry based approaches became possible, analytical sensitivity constraints meant that it was impossible to "fill the gaps" in understanding how the subcellular heterogeneity was generated. Lipid remodelling, post-synthesis was likely and possibly multiple sites of synthesis with differently available substrate pools together with possible differential transport phenomena within cells, but radiolabelling was too insensitive to provide a comprehensive picture and no technique provided precise answers.

\subsection{Compartments and organelles}

One example of the power of stable isotope labelling in defining parameters of subcellular organisation of PtdCho synthesis is provided by work with cells over-expressing an enzyme of the pathway. In bulk mammalian PtdCho membrane synthesis, the terminal step of the CDPcholine pathway, which catalyses the joining of diacylglycerol to an activated choline molecule, CDPcholine, is a CDPcholine 1,2diacylglycerol cholinephosphotransferase which is expressed in two isozymes CEPT1 and CPT1 [18]. When transfected into CHO-K1 cells, the human enzymes show topologically distinct locations within the extranuclear compartment with the former co-located with the ER/nuclear envelope and the latter colocalising with Golgi bodies [18]. Each isoform activity within the cell contributes around 50\% of bulk synthesis although distinct PtdCho biosynthesis does occur in lesser amounts at other cellular locations (Fig. 4). The endonuclear compartment is one distinct location of de novo PtdCho synthesis but neurite extensions also show a small autonomous PtdCho synthetic capacity when synthesising membrane for neurite extension [10].

Analyses of purified CEPT1 and CPT1 in vitro shows a degree of molecular selectivity for diacylglycerol species used for PtdCho synthesis de novo [18] but does not inform any molecular selectivity

\section{SITES OF MAMMALIAN CELL PHOSPHATIDYLCHOLINE SYNTHESIS}

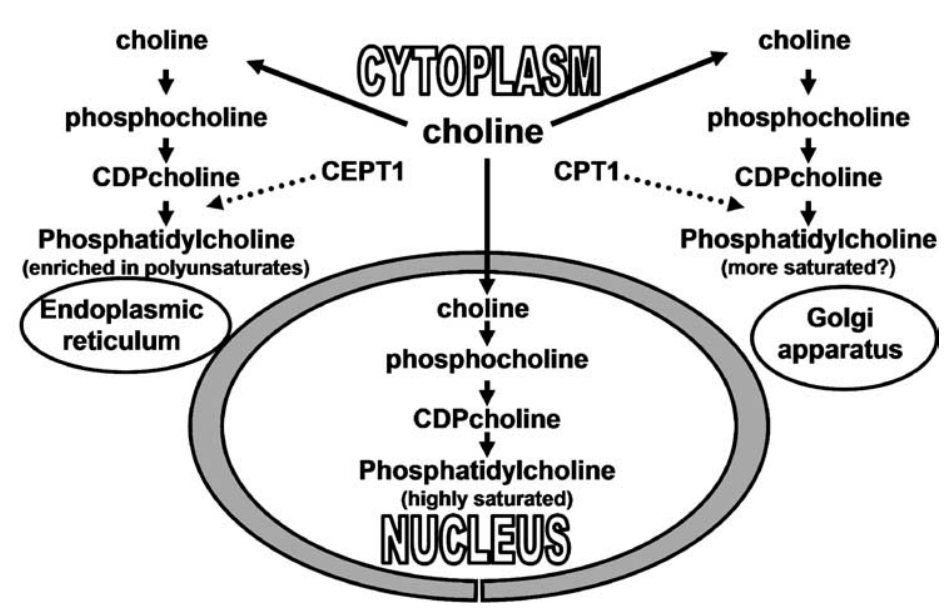

Fig. 4. Sites of phosphatidylcholine biosynthesis in mammalian cells. Bulk membrane PtdCho is synthesised at both the endoplasmic reticulum and the Golgi [18] in addition to smaller quantities synthesised inside cell nuclei [20,21,24]. The final transformation of CDPcholine to PtdCho by linkage with diacylglycerol is achieved by one of two enzymes, CEPT1 - located predominantly with the endoplasmic reticulum and nuclear envelope, and CPT1 predominantly associated with the Golgi. The corresponding enzyme inside the nuclear matrix has not been reported but may be CEPT1 as we have observed PtdEtn synthetic capacity in these fractions (unpublished data). 
which may exist in situ where the biosynthetic pathway is presented with a choice of diacylglycerol species. Labelling cells with choline- $d_{9}$ and analysing its subcellular flux can potentially reveal details of molecular specificity. For subcellular compartments which can be isolated after labelling such as the endonuclear matrix $[20,21,24]$ - (discussed below) time courses for the acquisition and remodelling newly synthesised PtdCho are facile. Within the extranuclear compartment, however, isolation of endoplasmic reticulum/nuclear envelope fractions that are free of Golgi contamination or vice versa is technically more difficult. An indirect alternative route to evaluating molecular specificity is offered by the ability to over express cholinephosphotransferases. Over expression of hCEPT1 in CHO-K1 cells under control of a TET-ON promoter increases cholinephosphotransferase activity 20 fold [18] without changing the rate of whole cell PtdCho accretion. Consequently, when cells are labelled with choline- $d_{9}$ $24 \mathrm{~h}$ after doxycycline induction, any whole cell change in PtdCho composition and patterns of synthesis will likely reflect a shift in diacylglycerol species preference of CEPT1 versus CPT1.

Such a difference was found by our investigations [22] and revealed an apparent and progressive in situ preference for incorporation of arachidonic acid $(20: 4)$ containing diacylglycerols over $3 \mathrm{~h}$. This was consistent either with a molecular specificity for these species or a subcellular enrichment of these species within the endoplasmic reticulum/nuclear envelope. Further organisational insight was provided by observation that the increased flux through the CEPT1 was likely countered by increases in both phospholipase $\mathrm{A}_{1}$ and phospholipase $\mathrm{A}_{2}$ activities as assessed by analyses of flux of the choline- $d_{9}$ through the whole cell lysoPtdCho pools.

The most extensively described use of dynamic lipidomic methodologies to elucidate PtdCho metabolism within an organelle has been undertaken within the nuclear matrix [19-21,24]. We have used choline- $d_{9}$ supplementation to confirm that the CDPcholine pathway enzymes identified by activity measurements in isolated nuclei form part of an autonomous endonuclear PtdCho synthesis pathway in a range of mammalian cells $[20,21,24]$. Moreover tracking the flux of choline- $d_{9}$ through cholinecontaining phospholipids from 10 minutes labelling for up to $24 \mathrm{~h}$ we were able to demonstrate that the molecular species composition of the endonuclear pool, in contrast to whole cell PtdCho is progressively remodelled to more saturated moieties. These studies provide both topological information concerning the organisation of subcellular PtdCho synthesis and mechanistic insights into compartmental remodelling.

\subsection{Whole cells}

The first reported use of choline- $d_{9}$ to characterise PtdCho synthesis in whole cells came from Cui's group in Wake Forest in 1999 [14]. Employment of this stable isotope in conjunction with ethanolamine$d_{4}$ enabled simultaneous determination of, and discrimination between, the two pathways of de novo PtdCho biosynthesis in hepatocytes. In addition to providing proof of concept, this pioneering study confirmed that the molecular specificity of polyunsaturated PtdCho for export from the liver via lipoprotein is predominantly attributable to the $\mathrm{N}$-methylation pathway of conversion of phosphatidylethanolamine (PtdEtn) to PtdCho rather than the CDPcholine pathway. While significantly active N-methylation pathway is confined to relatively few mammalian tissues, a series of papers using similar methodological approaches in Saccharomyces cerevisiae has recently showed that CDPcholine and N-methylation pathways here also produce a distinctly different profile of molecular species [4-6]. These authors used choline- $d_{13}$ and methyl- $d_{3}$ methionine to distinguish between the pathways although the approach is essentially identical. The precursor scans in these cases utilised $m / z 184^{+}, 193^{+}$and $197^{+}$.

Stable isotope labelling of CDPcholine and N-methylation pathways are also of interest in the malaria parasite Plasmodium falciparum where modifications of the deuterium labelling protocols described 
previously have been recently automated using fixed timepoint comparisons to simplify and to improve throughput rates [15]. Unlike the other studies described here, these studies essentially provided a rapid method for quantifying the relative contributions of CDPcholine and N-methylation pathways to whole cell PtdCho production but without the resort to the intricacies of molecular species evaluations.

Differentiated, non-proliferating cells in vivo operate homeostatic mechanisms which maintain phenotypic characteristics including defined molecular species compositions for their PtdCho which do not depend upon nutrient supply. Human venous blood samples, incubated with choline- $d_{9}$ for $3 \mathrm{~h}$ followed by separation and characterisation of the neutrophil and lymphocyte subpopulations of the white cells, revealed a different, distinct acquisition pattern and tightly regulated PtdCho profile for each [28]. This in turn may be related to functional requirements in vivo.

\subsection{Whole organisms}

The logical extrapolation of an ability to probe PtdCho cellular metabolism with choline- $d_{9}$ is to extend such studies to whole organisms. Notwithstanding the studies with yeast and plasmodium mentioned previously, the integrated nature of multicellular whole organism lipid metabolism provides immense scope for augmentation of choline- $d_{9}$ labelling studies at the cellular level. Investigation of PtdCho export and transport phenomena between organs and tissues, extracellular contributions to PtdCho turnover and uptake and recycling phenomena are all amenable to investigation.

One caveat is necessary when considering whole body metabolism of choline- $d_{9}$ however. Cui's group reported that choline- $d_{9}$ was subject in part to degradation via the betaine pathway which acts to retain methyl groups from choline ingested in excess of an organism's needs [13]. Deuterated methyl group salvage, via incorporation into s-adenosyl methionine, provides a route for ultimate introduction into PtdCho synthesised by the N-methylation pathway [13]. Accordingly, in whole animals labelled with choline- $d_{9}$, the progressive appearance of precursors of $\mathrm{m} / z 187^{+}$and $190^{+}$fragments reveal liver PtdCho molecules synthesized via N-methylation. Moreover, prolonged incubation times combined with PtdCho transport and turnover processes may result in distal tissues acquiring these labelled headgroups. Studies with mice injected with both choline- $d_{9}$ to track PtdCho and myo inositol- $d_{6}$ to track phosphatidylinositol (PtdIns) have been undertaken by our group although only the liver PtdIns synthesis and composition data has yet been published in preliminary form [27] while PtdCho data from, liver and plasma has only been presented in abstract form [http://www.biochemsoctrans.org/bst/BS2004/ BS2004R109.pdf] with full papers still in preparation.

Of those organs whose accessibility makes then suitable for study in situ, the lung has proved particularly important and clinically relevant. In phospholipid terms, over and above the PtdCho synthesis necessary for normal cell growth and repair, the lung synthesises and secretes into the alveolar space significant amounts of dipalmitoyl PtdCho $(16: 0 / 16: 0)$ the major surface active phospholipid component of pulmonary surfactant [1]. Removal of the ethical constraints that would preclude labelling humans with radionuclides, has also allowed whole body assessment of the effect of venous infusion of choline$d_{9}$ over several hours into healthy volunteers [3]. This study has reported choline- $d_{9}$ incorporations into pulmonary surfactant PtdCho recovered from induced sputum. Surfactant PtdCho synthesis rates, progressive remodelling from palmitoyloleoyl PtdCho to dipalmitoyl PtdCho and turnover/equilibration times were all calculated from data obtained.

Developmental immaturity of the mammalian lung is characterised by a lack of stored disaturated PtdCho [23] and reduced capacity to mobilise surfactant of the appropriate molecular species composition. Neonatal Respiratory Distress Syndrome (NRDS) has its origins in compromised surfactant function 
and an understanding of the metabolic processes underlying a particular patient's clinical presentation may aid effective therapeutic intervention. Stable isotope labelling of ventilated infant lungs via intratracheal administration is relatively easy and has been undertaken using both deuterated and $\left[{ }^{13} \mathrm{C}\right]$ labelled PtdCho precursors $[11,12,34]$. However, these latter studies while informative have not yet extended to the use of ESI-MS/MS characterisation of material recovered from tracheal aspirates and bronchoalveolar lavage. They have relied instead upon gas chromatography/mass spectrometry or gas chromatography/combustion/isotope ratio mass spectrometry. It is likely that considerably more information will flow from these types of analyses in the near future.

\section{Summary}

The increasing use of stable isotope labelling in conjunction with ESI-MS/MS analysis of phospholipids has thrust dynamic lipidomics into the centre stage of lipid metabolism. This review shows some of the uses to which the methodology has been applied but has only touched the surface of potential applications and the scope for further work is immense. In addition to qualitative and quantitative measurements of PtdCho molecular species metabolism, for example, a whole untapped field of TOF mass spectrometry-based secondary ion imaging opportunities exist with analyses of $m / z 184^{+}$ions allowing the topological identification of PtdCho molecules in membrane scanning [26]. In future it is likely that complementary imaging which incorporates scanning for $m / z 193^{+}$ions following choline- $d_{9}$ labelling may enable "visualisation" of the spatial pattern of membrane acquisition of newly synthesised PtdCho molecules.

\section{References}

[1] J.J. Batenburg and H.P. Haagsman, The lipids of pulmonary surfactant: dynamics and interactions with proteins, Prog. Lipid Res. 37 (1998), 235-276.

[2] W. Bernhard, M. Linck, H. Creutzburg, A.D. Postle, A. Arning, I. Martin-Carrera and K.F. Sewing, High-performance liquid chromatographic analysis of phospholipids from different sources with combined fluorescence and ultraviolet detection, Anal. Biochem. 220 (1994), 172-180.

[3] W. Bernhard, C.J. Pynn, A. Jaworski, G.A. Rau, J.M. Hohlfeld, J. Freihorst, C.F. Poets, D. Stoll and A.D. Postle, Mass spectrometric analysis of surfactant metabolism in human volunteers using deuteriated choline, Am. J. Respir. Crit. Care Med. 170 (2004), 54-58.

[4] H.A. Boumann, P.T. Chin, A.J. Heck, B. de Kruijff and A.I. de Kroon, The yeast phospholipid N-methyltransferases catalyzing the synthesis of phosphatidylcholine preferentially convert diC16:1 substrates both in vivo and in vitro, J. Biol. Chem. 279 (2004), 40314-41319.

[5] H.A. Boumann, M.J. Damen, C. Versluis, A.J. Heck, B. de Kruijff and A.I. de Kroon, The two biosynthetic routes leading to phosphatidylcholine in yeast produce different sets of molecular species. Evidence for lipid remodeling, Biochemistry 42 (2003), 3054-3059.

[6] H.A. Boumann, B. de Kruijff, A.J. Heck and A.I. de Kroon, The selective utilization of substrates in vivo by the phosphatidylethanolamine and phosphatidylcholine biosynthetic enzymes Epot1p and Cpt1p in yeast, FEBS Lett. 569 (2004), 173-177.

[7] G.C. Burdge, A.N. Hunt and A.D. Postle, Mechanisms of hepatic phosphatidylcholine synthesis in adult rat: effects of pregnancy, Biochem. J. 303 (1994), 941-947.

[8] G.C. Burdge, F.J. Kelly and A.D. Postle, Synthesis of phosphatidylcholine in guinea-pig fetal lung involves acyl remodelling and differential turnover of individual molecular species, Biochim. Biophys. Acta 1166 (1993), 251-257.

[9] P.A. Caesar, S.J. Wilson, I.C.S. Normand and A.D. Postle, A comparison of the specificity of phosphatidylcholine synthesis by human fetal lung maintained in either organ or organotypic culture, Biochem. J. 253 (1988), 451-457.

[10] J.M. Carter, K.A. Waite, R.B. Campenot, J.E. Vance and D.E. Vance, Enhanced expression and activation of CTP:phosphocholine cytidylyltransferase beta2 during neurite outgrowth, J. Biol. Chem. 278 (2003), 44988-44994. 
[11] P.E. Cogo, L.J. Zimmermann, L. Meneghini, N. Mainini, L. Bordignon, V. Suma, M.Buffo and V.P. Carnielli, Pulmonary surfactant disaturated-phosphatidylcholine (DSPC) turnover and pool size in newborn infants with congenital diaphragmatic hernia (CDH), Pediatr. Res. 54 (2003), 653-658.

[12] P.E. Cogo, L.J. Zimmermann, G. Verlato, P. Midrio, A. Gucciardi, C. Ori and V.P. Carnielli, A dual stable isotope tracer method for the measurement of surfactant disaturated-phosphatidylcholine net synthesis in infants with congenital diaphragmatic hernia, Pediatr. Res. 56 (2004), 184-190.

[13] C.J. DeLong, A.M. Hicks and Z. Cui, Disruption of choline methyl group donation for phosphatidylethanolamine methylation in hepatocarcinoma cells, J. Biol. Chem. 277 (2002), 17217-17225.

[14] C.J. DeLong, Y.J. Shen, M.J. Thomas and Z. Cui, Molecular distinction of phosphatidylcholine synthesis between the CDP-choline pathway and the phosphatidylethanolamine methylation pathway, J. Biol. Chem. 274 (1999), 29683-29688.

[15] C. Enjalbal, R. Roggero, R. Cerdan, J. Martinez, H.Vial and J.L. Aubagnac, Automated monitoring of phosphatidylcholine biosyntheses in plasmodium falciparum by electrospray ionization mass spectrometry through stable isotope labeling experiments, Anal. Chem. 76 (2004), 4515-4521.

[16] M. Gobley, Examen comparatif du jaune d'oeufe et de al matiere cerebrale, J. Pharm. Chim. 11 (1847), 409.

[17] X. Han and R.W. Gross, Global analyses of cellular lipidomes directly from crude extracts of biological samples by ESI mass spectrometry: a bridge to lipidomics, J. Lipid Res. 44 (2003), 1071-1079.

[18] A.L. Henneberry, M.M. Wright and C.R. McMaster, The major sites of cellular phospholipid synthesis and molecular determinants of fatty acid and lipid head group specificity, Mol. Biol. Cell. 13 (2002), 3148-3161.

[19] A.N. Hunt, J.G. Alb, G. Koster, A.D. Postle and V.A. Bankaitis, Use of mass spectrometry-based lipidomics to probe PITP $\alpha$ (phosphatidylinositol transfer protein alpha) function inside the nuclei of PITP $\alpha^{+/+}$and PITP $\alpha^{-/-}$cells, Biochem. Soc. Trans. 32 (2004), 1063-1065.

[20] A.N. Hunt, G.T. Clark, G.S. Attard and A.D. Postle, Highly saturated endonuclear phosphatidylcholine is synthesized in situ and colocated with CDP-choline pathway enzymes, J. Biol. Chem. 276 (2001), 8492-8499.

[21] A.N. Hunt, G.T. Clark, J.R. Neale and A.D. Postle, A comparison of the molecular specificities of whole cell and endonuclear phosphatidylcholine synthesis, FEBS Lett. 530 (2002), 89-93.

[22] A.N. Hunt, H.C. Fenn, G.T. Clark, M.M. Wright, A.D. Postle and C.R. McMaster, Lipidomic analysis of the molecular specificity of a cholinephosphotransferase in situ, Biochem. Soc. Trans. 32 (2004), 1060-1062.

[23] A.N. Hunt, F.J. Kelly and A.D. Postle, Developmental variation in whole human lung phosphatidylcholine molecular species: a comparison with guinea pig and rat, Early Hum. Dev. 25 (1991), 157-171.

[24] A.N. Hunt and A.D. Postle, Phosphatidylcholine biosynthesis inside the nucleus: is it involved in regulating cell proliferation?, Adv. Enz. Regul. 44 (2004), 173-186.

[25] A.N. Hunt, A.J. Skippen, G. Koster, A.D. Postle and S. Cockcroft, Acyl chain-based molecular selectivity for HL60 cellular phosphatidylinositol and of phosphatidylcholine by phosphatidylinositol transfer protein alpha, Biochim. Biophys. Acta 1686 (2004), 50-60.

[26] S.G. Ostrowski, C.T. Van Bell, N. Winograd and A.G. Ewing, Mass spectrometric imaging of highly curved membranes during Tetrahymena mating, Science 305 (2004), 71-73.

[27] A.D. Postle, H. Dombrowsky, H. Clarke, C.J. Pynn, G. Koster and A.N. Hunt, Mass spectroscopic analysis of phosphatidylinositol synthesis using 6-deuteriated-myo-inositol: comparison of the molecular specificities and acyl remodelling mechanisms in mouse tissues and cultured cells, Biochem. Soc. Trans. 32 (2004), 1057-1059.

[28] A.D. Postle, J. Madden, G.T. Clark and S.M. Wright, Electrospray ionization mass spectrometry analysis of differential turnover of phosphatidylcholine by human blood leukocytes, Phys. Chem. Chem. Phys. 6 (2004), 1018-1021.

[29] A.D. Postle, Method for the sensitive analysis of individual molecular species of phosphatidylcholine by high-performance liquid chromatography using post-column fluorescence detection, J. Chromatogr. 415 (1987), 241-251.

[30] M. Pulfer and R.C. Murphy, Electrospray mass spectrometry of phospholipids, Mass Spectrom. Rev. 22 (2003), $332-364$.

[31] S. Ruggierri, R. Roblin and P.H. Black, Lipids of whole cells and plasma membrane fractions from Balb/c3T3, SV3T3, and concanavalin A-selected revertant cells, J. Lipid Res. 20 (1979), 760-771.

[32] K. Tran, Y. Wang, C.J. DeLong and Z. Cui, The assembly of very low density lipoproteins in rat hepatoma McA-RH7777 cells is inhibited by phospholipase A2 antagonists, J. Biol. Chem. 275 (2000), 25023-25030.

[33] J.T. Venkatraman, T. Toohey and M.T. Clandinin, Does a threshold for the effect of dietary omega-3 fatty acids on the fatty acid composition of nuclear envelope phospholipids exist?, Lipids 27 (1992), 94-97.

[34] G. Verlato, P.E. Cogo, E. Benetti, S. Gomirato, A. Gucciardi and V.P. Carnielli, Kinetics of surfactant in respiratory diseases of the newborn infant, J. Matern. Fetal Neonatal Med. 16(Suppl. 2) (2004), 21-24.

[35] S.D. Williams, F.F. Hsu, D.A. Ford, Electrospray ionization mass spectrometry analyses of nuclear membrane phospholipid loss after reperfusion of ischemic myocardium, J. Lipid Res. 41 (2000), 1585-1595. 


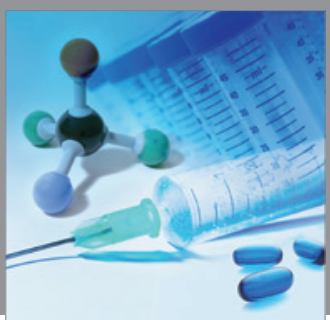

International Journal of

Medicinal Chemistry

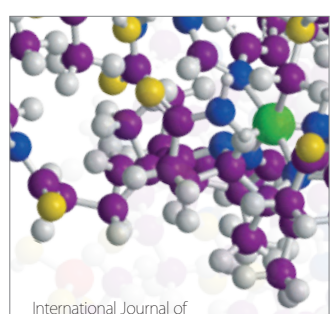

Carbohydrate Chemistry

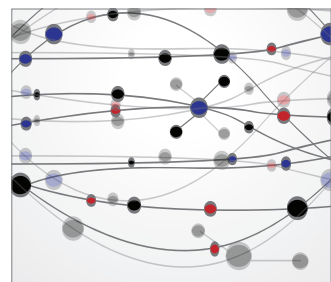

The Scientific World Journal
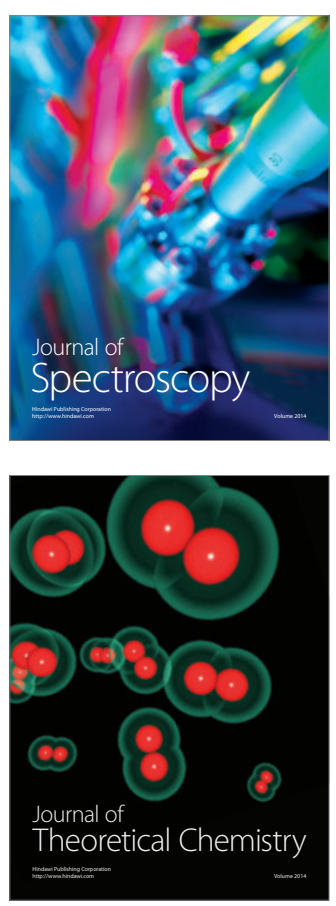
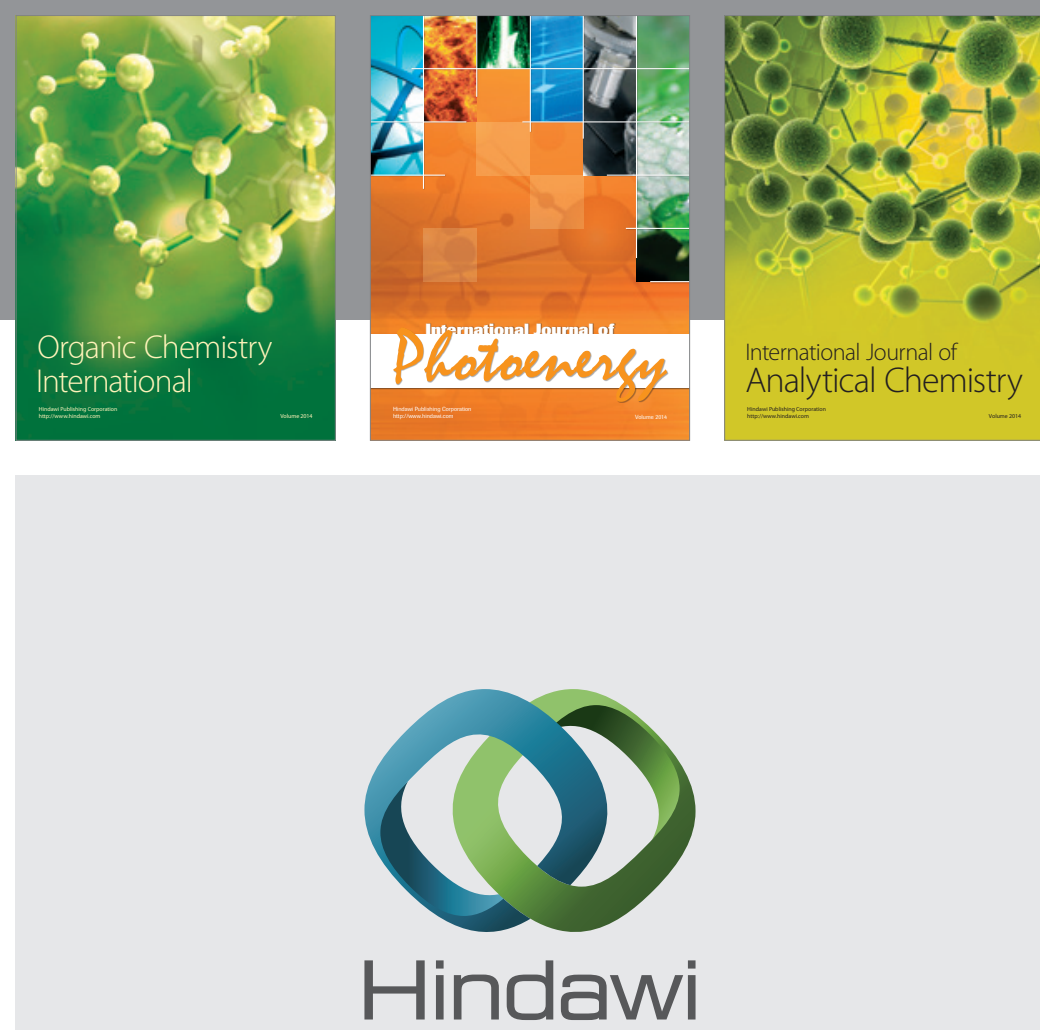

Submit your manuscripts at

http://www.hindawi.com
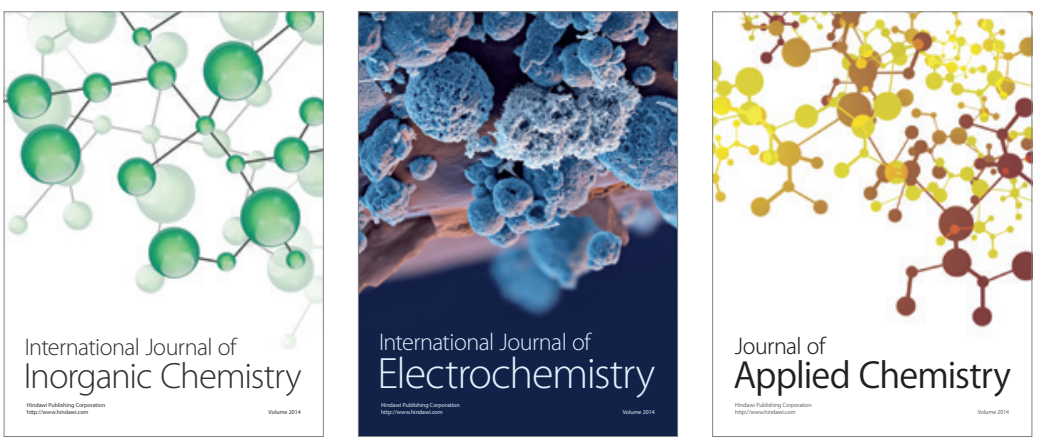

Journal of

Applied Chemistry
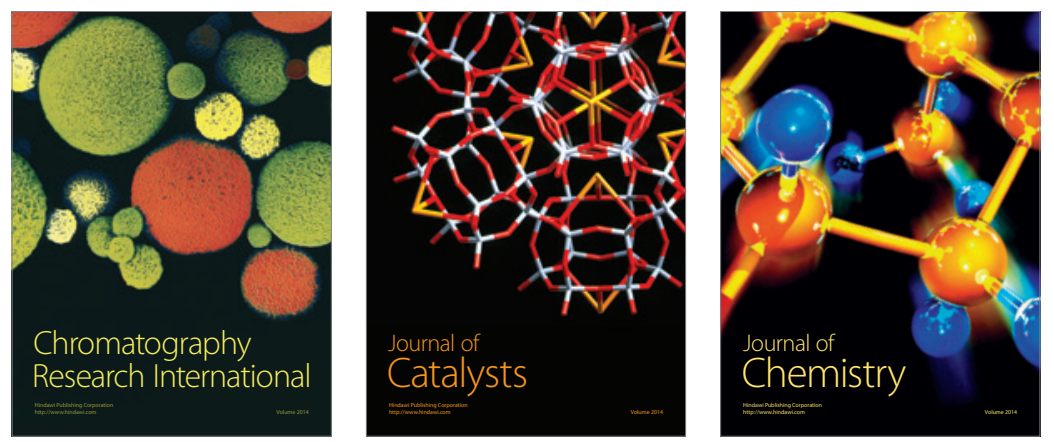
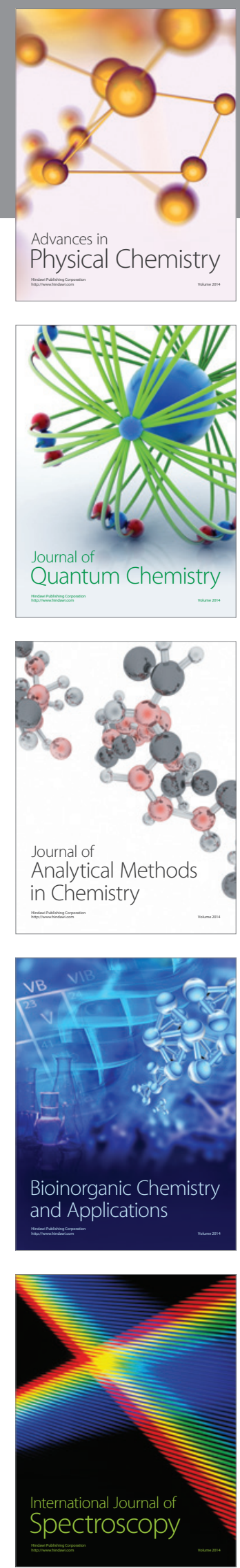